\title{
Neutrino Tests of General and Special Relativity
}

\author{
C. N. Leungf \\ Department of Physics and Astronomy, University of Delaware \\ Newark, DE 19716, U.S.A.
}

\begin{abstract}
We review the status of testing the principle of equivalence and Lorentz invariance from atmospheric and solar neutrino experiments.
\end{abstract}

\footnotetext{
${ }^{1}$ Talk presented at the Nufact'99 Workshop, Lyon, France, July 5-9, 1999.
} 
Let us begin by asking the question: do neutrinos obey the principle of equivalence? The answer must of course come from experiments. For ordinary matter, the most sensitive test of the equivalence principle comes from torsion balance experiments which measure the gravitational acceleration of macroscopic bodies. The current best limit [1] is $10^{-12}$, i.e., all macroscopic bodies experience the same gravitational acceleration to an accuracy of one part in $10^{12}$. Surely torsion balance experiments are not applicable to neutrinos. So the question is: what types of experiments are suitable for testing if neutrinos obey the principle of equivalence?

Suppose the neutrinos violate the principle of equivalence. A consequence will be that different neutrino types (i.e., different neutrino gravitational eigenstates) will couple to gravity with a slightly different strength. Suppose the neutrino weak interaction eigenstates are not the same as their gravitational interaction eigenstates, but are linear superpositions of them. Then, as a neutrino of definite flavour (e.g., $\nu_{\mu}$ ) travels through a gravitational field, the gravitational components of the flavour neutrino will develop different dynamical phases, which will result in neutrino flavour oscillations. In other words, neutrino oscillation experiments provide a laboratory to test the equivalence principle for neutrinos 2, 3].

To parametrize the probabilities for neutrino oscillations arising from a violation of the equivalence principle (VEP), we follow the formalism in Ref. [1] where the general equation governing neutrino flavour evolutions is found to be

$$
\begin{aligned}
i \frac{d}{d t}\left(\begin{array}{c}
\nu_{e} \\
\nu_{\mu}
\end{array}\right) & =\left\{\frac{\Delta m^{2}}{4 E}\left[\begin{array}{cc}
-\cos 2 \theta_{M} & \sin 2 \theta_{M} \\
\sin 2 \theta_{M} & \cos 2 \theta_{M}
\end{array}\right]\right. \\
& +E|\phi(r)| \Delta f\left[\begin{array}{cc}
-\cos 2 \theta_{G} & e^{-i \beta} \sin 2 \theta_{G} \\
e^{i \beta} \sin 2 \theta_{G} & \cos 2 \theta_{G}
\end{array}\right] \\
& \left.+\frac{G_{F} N_{e}}{\sqrt{2}}\left[\begin{array}{cc}
1 & 0 \\
0 & -1
\end{array}\right]\right\}\left(\begin{array}{l}
\nu_{e} \\
\nu_{\mu}
\end{array}\right) .
\end{aligned}
$$

We have assumed two neutrino flavours $\left(\nu_{e}\right.$ and $\left.\nu_{\mu}\right)$ for simplicity. The first and third line on the right-hand side of Eq.(1) are familiar from the usual studies of neutrino oscillations. The first line describes flavour mixing arising from nondegenerate neutrino vacuum masses [5]. Here $E$ denotes the neutrino's energy, $\Delta m^{2} \equiv m_{2}^{2}-m_{1}^{2}$ is the neutrino mass-squared difference, 
and $\theta_{M}$ is the mixing angle between the weak interaction eigenstates and the mass eigenstates of the neutrinos. The third line describes the matter effects on neutrino flavour evolutions [6]. Here $G_{F}$ is the Fermi constant and $N_{e}$ is the electron density in matter. To study neutrino oscillations in vacuum, simply set $N_{e}=0$. The second line on the right-hand side of Eq.(11) describes neutrino flavour mixing arising from VEP. Here $\phi(r)$, which satisfies the boundary condition $\phi(r \rightarrow \infty) \rightarrow 0$, is the gravitational potential through which the neutrinos propagate, $\theta_{G}$ is the mixing angle between the weak interaction eigenstates and the gravitational interaction eigenstates of the neutrinos, $\beta$ is a phase factor which comes about because we have mixing among three generally distinct sets of neutrino eigenstates: weak, gravitational, and mass. Finally, $\Delta f$ is a measure of VEP defined as $\Delta f \equiv f_{2}-f_{1}$, where $f_{1,2}$ are parameters introduced in the linearized neutrino-gravity interaction Lagrangian density [7],

$$
\mathcal{L}_{\text {int }}=\frac{i f_{j}}{4} \sqrt{8 \pi G_{N}} h^{\alpha \beta}\left[\overline{\nu_{j}} \gamma_{\alpha} \partial_{\beta} \nu_{j}-\left(\partial_{\alpha} \overline{\nu_{j}}\right) \gamma_{\beta} \nu_{j}\right], \quad j=1,2
$$

to gauge the deviation from universal coupling to gravity. Here $\nu_{1,2}$ denote the neutrino gravitational eigenstates, $h^{\alpha \beta}$ is the background gravitational field, and $G_{N}$ is the Newton gravitational constant. Einstein's theory of general relativity predicts $f_{1}=f_{2}=1$. VEP occurs when $f_{1} \neq f_{2}$.

It can be concluded from Eq.(1) that

1. VEP leads to neutrino flavour oscillations even if the neutrinos are massless or degenerate (in this case the phase factor $\beta$ can be eliminated by redefinition of the neutrino fields);

2. neutrino oscillation tests of the principle of equivalence involve two parameters: $\Delta f$ and $\theta_{G}$; it is also necessary to know the value of the local gravitational potential;

3. VEP oscillations have a different energy dependence from oscillations due to nondegenerate neutrino masses.

The dependence of VEP oscillations on $\phi(r)$ is a consequence of violating the principle of equivalence. It is also a source of uncertainty. Table 1 in Ref. [4] shows that our local value of $|\phi|$ spans from $6 \times 10^{-10}$ for the Earth's gravitational potential to about $3 \times 10^{-5}$ for the gravitational potential due 
to our local supercluster [8]. Its value may be even bigger if the contributions from more distant sources can be estimated. Since $\phi$ appears to be dominated by distant sources, its value varies little over a typical neutrino path length for earthbound experiments and may thus be treated as a constant. It is then convenient to treat $|\phi| \Delta f$ as a single parameter in phenomenological analyses.

In the constant $\phi$ approximation, the VEP flavour transition probability has the familiar form $\left(\Delta m^{2}\right.$ is assumed to be zero here):

$$
P\left(\nu_{e} \rightarrow \nu_{\mu}\right)=\sin ^{2}\left(2 \theta_{G}\right) \sin ^{2}\left(\frac{\pi L}{\lambda_{G}}\right)
$$

where $L$ is the neutrino path length and $\lambda_{G}$ is the oscillation length given by

$$
\lambda_{G}=\frac{\pi}{E|\phi| \Delta f}=6.2 \mathrm{~km}\left(\frac{10^{-19}}{|\phi| \Delta f}\right)\left(\frac{1 \mathrm{GeV}}{E}\right) .
$$

If we compare this with the well-known vacuum oscillation probability due to a neutrino mass difference, we see that the two cases are related by the formal connection,

$$
\frac{\Delta m^{2}}{4 E} \rightarrow E|\phi| \Delta f \quad \text { and } \quad \theta_{M} \rightarrow \theta_{G}
$$

This connection can also be gleaned from Eq.(1). In contrast to the case of flavour oscillations induced by a neutrino mass difference where the oscillation length increases with the neutrino's energy, VEP oscillations are characterized by an oscillation length that decreases with increasing neutrino energy. The two mechanisms may therefore be distinguished by measuring the neutrino energy spectrum 9, 10, 11, 12]. Note also that VEP oscillations will be more prominent in experiments with a large $E \cdot L$, i.e., high energy neutrinos and/or long path lengths, which is ideal for the neutrino factory experiments discussed in this workshop.

In a certain class of string theories, VEP may arise through interactions with a massless dilaton field [13]. This type of equivalence principle violation may also lead to neutrino oscillations, but with an energy dependence which is the same as in oscillations due to nondegenerate neutrino masses [14]. It is therefore difficult to distinguish this type of VEP oscillations from the mass mixing oscillations, although one can still use data from oscillation 
experiments to constrain the relevant neutrino-dilaton couplings[14]. We shall not consider further VEP oscillations from string theory, but will focus on the VEP oscillations from Eq.(1), which share the same distinctive energy dependence 15 with neutrino oscillations arising from a possible breakdown of Lorentz invariance 16.

If Lorentz invariance is violated, different (massless) neutrino species may have different maximum attainable speeds which are close to but not necessarily equal to $c$. Neutrino oscillations can occur if the neutrino flavour eigenstates are linear superpositions of their velocity eigenstates, defined to be the energy eigenstates at infinite momentum, with the probability (for two-neutrino mixing) [16]

$$
P\left(\nu_{e} \rightarrow \nu_{\mu}\right)=\sin ^{2}\left(2 \theta_{v}\right) \sin ^{2}\left(\frac{\pi L}{\lambda_{v}}\right)
$$

where $L$ is the neutrino path length, $\theta_{v}$ is the mixing angle between the weak interaction eigenstates and the velocity eigenstates of the neutrinos, and $\lambda_{v}$ is the oscillation length given by

$$
\lambda_{v}=\frac{2 \pi}{E \Delta v}=1.24 \mathrm{~km}\left(\frac{10^{-18}}{\Delta v}\right)\left(\frac{1 \mathrm{GeV}}{E}\right) .
$$

Here $\Delta v=v_{2}-v_{1}$ is the difference between the speeds of the two neutrino velocity eigenstates. Comparing Eqs.(6) and (7) with Eqs.(3) and (4), the similarities between the VLI (violation of Lorentz invariance) oscillations and VEP oscillations (for constant $\phi$ ) are obvious.

Eqs.(4) and (7) indicate the sensitivity we can expect for VEP and VLI tests from neutrino oscillation experiments. Assuming maximal mixing, there will be appreciable flavour transitions if $\pi L / \lambda_{G, v} \sim O(1)$. For atmospheric neutrinos, $E \sim\left(0.1-10^{3}\right) \mathrm{GeV}$ and $L \sim\left(20-10^{4}\right) \mathrm{km}$, hence $|\phi| \Delta f$ (or $\left.\Delta v / 2\right)$ in the range $\left(10^{-26}-10^{-19}\right)$ can be probed. For solar neutrinos, $E \sim(0.1-10)$ $\mathrm{MeV}$ and, for vacuum oscillations, $L \sim 10^{8} \mathrm{~km}$, hence $|\phi| \Delta f($ or $\Delta v / 2)$ in the range $\left(10^{-25}-10^{-23}\right)$ can be probed. For MSW solution to the solar neutrino problem, the solar gravitational potential, which is about $1.7 \times 10^{-5}$ at the solar core and decreases to about $2 \times 10^{-6}$ at the surface of the Sun, plays a significant role and the constant $\phi$ approximation is not as good here. Nevertheless we can obtain an order-of-magnitude estimate for the sensitivity limit : $|\phi| \Delta f($ or $\Delta v / 2) \sim\left(10^{-23}-10^{-22}\right)$, by letting $L=7 \times 10^{5} \mathrm{~km}$, the 
mean solar radius (see Fig. 2 in Ref. [10] for a more accurate estimate). For long-baseline experiments such as those envisaged for future neutrino factories, $E \sim(10-100) \mathrm{GeV}$ and $L \sim\left(\right.$ few $\times 10^{2}-$ few $\left.\times 10^{3}\right) \mathrm{km}$, hence a sensitivity of $\left(10^{-25}-10^{-23}\right)$ for $|\phi| \Delta f$ (or $\left.\Delta v / 2\right)$ can be reached. These estimates demonstrate that neutrino oscillations can provide a very sensitive test for the fundamental principles of general and special relativity.

We now confront VEP and VLI with experiments. Because of the limited time, we shall consider only atmospheric and solar neutrino experiments.

Positive evidence for $\nu_{\mu} \rightarrow \nu_{\tau}$ transitions has been established by the Super-Kamiokande (SK) Collaboration[17]. An analysis [18 of the 535 days of SK data on sub-GeV and multi-GeV events found that VEP and VLI oscillations were consistent with the data, although the $\chi^{2}$ fit was not as good as the fit for mass mixing oscillations. However, later analyses [19, 20 that included more recent SK data 21] on upward-going muon events, which corresponded to atmospheric neutrinos with higher energies: $E$ up to $\sim 10^{3}$ $\mathrm{GeV}$, found that VEP and VLI oscillations were not compatible with the data. In particular, Fogli et al. [20], did a very thorough analysis of the VEP and VLI mechanisms with the SK data. They allowed a power-law energy dependence for the oscillation length: $\lambda \propto E^{-n}$, and found that, at $90 \%$ C.L., $n=-0.9 \pm 0.4$, which identified the mass mixing mechanism as the mechanism for the observed $\nu_{\mu} \rightarrow \nu_{\tau}$ transitions. VEP and VLI oscillations, which correspond to $n=1$, are clearly incompatible with the data. The possibility of both mass mixing and VEP (or VLI) mechanisms contributing to the flavour transitions, as described in Eq.(1), was also studied in Ref. 20. It was found that, for a wide range of parameter choices, including the VEP (or VLI) contributions did not improve the fit for the mass mixing mechanism, which allowed the authors to obtain the $90 \%$ C.L. upper bounds for the $\nu_{\mu}-\nu_{\tau}$ sector:

$$
|\Delta f|<10^{-19}, \quad|\Delta v|<6 \times 10^{-24},
$$

independent of the values of the corresponding mixing angles. The limit on $\Delta f$ is obtained by assuming $|\phi|=3 \times 10^{-5}$, the contribution from the local supercluster. This limit on VEP is seven orders of magnitude more stringent than the best limit from torsion balance experiments [1]. The limit on $\Delta v$ is also the most stringent limit on VLI to-date. Note finally that these bounds are consistent with the range of sensitivity discussed above and are within about an order of magnitude of the sensitivity limit for long-baseline 
experiments.

As constraining as the SK atmospheric neutrino data are, they only tell us about VEP and VLI in the $\nu_{\mu}-\nu_{\tau}$ sector. To study flavour transitions involving $\nu_{e}$, we turn to solar neutrino experiments. The observed solar neutrino deficit may be a result of long-wavelength neutrino vacuum oscillations or matter-enhanced flavour transitions (MSW effect) [6, 22] in the Sun. Based on the Standard Solar Model predictions in Ref. 223], it was found in Ref. [9] that only matter-enhanced VEP oscillations were compatible with the available solar neutrino data then. However, using the improved Standard Solar Model predictions 24] and the latest solar neutrino data 25, 26, 27, 28], and treating the ${ }^{8} \mathrm{~B}$ neutrino flux as a free parameter, a recent study [29] finds that long-wavelength VEP oscillations can also account for the solar neutrino deficit. The authors of Ref.[29] examined constraints from the observed solar neutrino rates and from the SK spectral data. They found that, for $\nu_{e} \rightarrow \nu_{\tau}$ transitions, the allowed parameter space lay in the region

$$
0.65 \leq \sin ^{2}\left(2 \theta_{G}\right) \leq 1.0 \text { and } 3.3 \times 10^{-20}<|\Delta f| \leq 3.3 \times 10^{-18}
$$

for VEP and

$$
0.65 \leq \sin ^{2}\left(2 \theta_{v}\right) \leq 1.0 \text { and } 2.0 \times 10^{-24}<|\Delta v| \leq 2.0 \times 10^{-22}
$$

for VLI. We have again used $|\phi|=3 \times 10^{-5}$ to obtain the limits on $\Delta f$ in (9). For $\nu_{e} \rightarrow \nu_{\mu}$ transitions, constraints [30] from the recent accelerator neutrino data from the CCFR Collaboration 31] reduce the allowed range for $|\Delta f|$ and $|\Delta v|$ to $3.3 \times 10^{-20}<|\Delta f|<6.7 \times 10^{-19}$ and $2.0 \times 10^{-24}<|\Delta v|<4.0 \times 10^{-23}$, for the same range of values for the corresponding mixing angle.

The VEP and VLI mechanisms also admit a MSW solution to the solar neutrino problem. One generally finds two disjointed allowed regions, one for small mixing angles and one for large mixings [4, 9, 10, 30, 32]. Figure 1, which is due to P. I. Krastev, shows the $99 \%$ confidence level allowed regions for $|\Delta f|$ and $\sin ^{2}\left(2 \theta_{G}\right)$ derived from comparing all available data on the solar neutrino rates, including the 825 days of SK data[28], with the Standard Solar Model predictions in Ref. 24]. Figure 1 is obtained by using only the solar gravitational potential for $\phi(r)$ in Eq.(1) to determine the neutrino flavour evolution. For the $\nu_{e} \rightarrow \nu_{\mu}$ channel, the allowed regions in Figure 1 are completely ruled out by the constraints from the CCFR data 30. For the $\nu_{e} \rightarrow \nu_{\tau}$ channel, only the large mixing angle region is incompatible with 
the CCFR dataf. The remaining small mixing region may be constrained by the SK data on the recoil-electron energy spectrum, although an analysis 32 based on earlier (504 days) SK data did not find such a constraint.

In conclusion, in addition to helping determine the masses and mixing angles of neutrinos, neutrino oscillation experiments constitute a very useful tool for testing the principle of equivalence and Lorentz invariance. Current solar and atmospheric neutrino data already provide very stringent limits. For the $\nu_{\mu}-\nu_{\tau}$ sector, atmospheric neutrino data imply that the principle of equivalence cannot be violated by more than one part in $10^{19}$ and Lorentz invariance cannot be violated by more than $6 \times 10^{-24}$. Solar neutrino data provide limits for both the $\nu_{e}-\nu_{\mu}$ and $\nu_{e}-\nu_{\tau}$ sectors. The exact limits depend on the assumption of either MSW oscillations or long-wavelength vacuum oscillations, with the long-wavelength solution imposing the stronger bounds. Future long-baseline experiments can help improve these limits with sufficiently long (thousands of kilometers) baselines and sufficiently high energy (hundreds of $\mathrm{GeV}$ ) neutrinos. A neutrino factory will be a big access for reaching these goals.

\section{Acknowledgement}

This work was supported in part by the U.S. Department of Energy under grant DE-FG02-84ER40163.

\section{References}

[1] V. B. Braginsky and V. I. Panov, Sov. Phys. JETP 34 (1972) 463. For a more recent experiment, see, e.g., B. R. Heckel, et al., Phys. Rev. Lett. 63 (1989) 2705.

[2] M. Gasperini, Phys. Rev. D 38 (1988) 2635; Phys. Rev. D 39 (1989) 3606.

\footnotetext{
${ }^{2}$ The authors of Ref. [30] claim that a three-neutrino mixing analysis rules out the MSW allowed regions completely, for both $\nu_{e} \rightarrow \nu_{\mu}$ and $\nu_{e} \rightarrow \nu_{\tau}$ transitions.
} 
[3] A. Halprin and C. N. Leung, Phys. Rev. Lett. 67 (1991) 1833.

[4] A. Halprin, C. N. Leung, and J. Pantaleone, Phys. Rev. D 53 (1996) 5365 .

[5] B. M. Pontecorvo, Zh. Eksp. Teor. Fiz. 34 (1958) 247 [Sov. Phys. JETP 7 (1958) 172]; Z. Maki, M. Nakagawa, and S. Sakata, Prog. Theor. Phys. 28 (1962) 870.

[6] L. Wolfenstein, Phys. Rev. D 17 (1978) 2369; Phys. Rev. D 20 (1979) 2634.

[7] D. G. Boulware and S. Deser, Ann. Phys. (N.Y.) 89 (1975) 193.

[8] I. R. Kenyon, Phys. Lett. B 237 (1990) 274.

[9] J. Pantaleone, A. Halprin, and C. N. Leung, Phys. Rev. D 47 (1993) R4199.

[10] J. N. Bahcall, P. I. Krastev, and C. N. Leung, Phys. Rev. D 52 (1995) 1770 .

[11] C. N. Leung and P. I. Krastev, in The Albuquerque Meeting, Proceedings of the 8th Meeting of the Division of Particles and Fields of the American Physical Society, ed. S. Seidel (World Scientific, Singapore, 1995), p.1544.

[12] H. Minakata and H. Nunokawa, Phys. Rev. D 51 (1995) 6625.

[13] T. Damour and A. M. Polyakov, Gen. Rel. Grav. 26 (1994) 1171; Nucl. Phys. B423 (1994) 532.

[14] A. Halprin and C. N. Leung, Phys. Lett. B 416 (1998) 361.

[15] S. L. Glashow, A. Halprin, P. I. Krastev, C. N. Leung, and J. Pantaleone, Phys. Rev. D 56 (1997) 2433.

[16] S. Coleman and S. L. Glashow, Phys. Lett. B 405 (1997) 249.

[17] Super-Kamiokande Collaboration, Y. Fukuda et al., Phys. Rev. Lett. 81 (1998) 1562; Phys. Lett. B 433 (1998) 9; Phys. Lett. B 436 (1998) 33. 
[18] R. Foot, C. N. Leung, and O. Yasuda, Phys. Lett. B 443 (1998) 185.

[19] P. Lipari and M. Lusignoli, Phys. Rev. D 60 (1999) 013003.

[20] G. L. Fogli, E. Lisi, A. Marrone, and G. Scioscia, Phys. Rev. D 60 (1999) 053006.

[21] Super-Kamiokande Collaboration, Y. Fukuda et al., Phys. Rev. Lett. 82 (1999) 2644.

[22] S. P. Mikheyev and A. Yu Smirnov, Yad. Fiz. 42 (1985) 1441 [Sov. J. Nucl. Phys. 42 (1985) 913].

[23] J. N. Bahcall and R. K. Ulrich, Rev. Mod. Phys. 60 (1988) 297; J. N. Bahcall and M. H. Pinsonneault, Rev. Mod. Phys. 64 (1992) 885.

[24] J. N. Bahcall, S. Basu, and M. H. Pinsonneault, Phys. Lett. B 433 (1998) 1.

[25] Homestake Collaboration, K. Lande et al., Astrophys. J. 496 (1998) 505.

[26] SAGE Collaboration, J. N. Abdurashitov et al., astro-ph/9907113.

[27] GALLEX Collaboration, W. Hampel et al., Phys. Lett. B 447 (1999) 127.

[28] Y. Suzuki for the Super-Kamiokande Collaboration, talk at the LeptonPhoton '99 Conference.

[29] A. M. Gago, H. Nunokawa, and R. Zukanovich Funchal, hepph/9909250.

[30] J. Pantaleone, T. K. Kuo, and S. W. Mansour, hep-ph/9907478.

[31] CCFR Collaboration, A. Romosan et al., Phys. Rev. Lett. 78 (1997) 2912; D. Naples et al., Phys. Rev. D 59 (1999) 031101.

[32] S. W. Mansour and T. K. Kuo, Phys. Rev. D 60 (1999) 097301. 